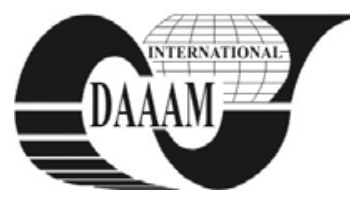

\title{
CALCULUS APPLICATIONS FOR DETERMINING THE TOTAL ELASTIC DISPLACEMENTS OF P-JOINTS
}

\author{
MARINESCU, D[an] A[ndrei] \& NICOLESCU, A[drian] F[lorin]
}

\begin{abstract}
Elastic displacements that occur inside P-joint have considerable impact on gantry robot's accuracy. For this purpose an original approach was made to evaluate the influence of total elastic displacement of prismatic joints on gantry robots volumetric accuracy. The authors developed a method based on custom made calculus applications and specific models developed with a special-made software program. First step of calculus method consists in determining the load on each guiding element, after, the elastic displacements appeared in guiding elements, are determined and the last step, consists in calculating the total elastic displacement of a P-joint. Combining results from each joint, the robot's positioning error induced by joints elastic displacements is determined.
\end{abstract}

Key words: industrial robots, accuracy, elastic displacements, numerical models

\section{INTRODUCTION}

The elastic displacements within joints have great impact on robots accuracy, approximately $70 \%$ of total positioning error is given by elastic displacements of joints.

In this paper the authors propose several analysis applications made for determining the elastic displacements of particular Gantry robot prismatic joints.

It is known that the guiding system of prismatic joints has the biggest contribution on totally joint's elastic deformation, practically, the joint's carriage is fully rigid compared with guiding elements.

In the present study, the authors analyzed the joints of a gantry robot manufactured by Gudel that uses for guiding a system based on cam-followers.

The mathematical model of a real gantry robot, according to Denavit-Hartenberg algorithm, in witch was added supplementary terms (terms that are expressing the elastic displacements, specific for a real robot), can be written as follows: (Nicolescu A. \& Stanciu M, 1996):

$$
T_{I R}^{\text {real }}=T_{I} T_{1}^{H D} T_{I I} \Delta_{1}^{J} \Delta_{1}^{S} T_{I I I} T_{I V} T_{2}^{H D} T_{V} \Delta_{2}^{J} \Delta_{2}^{S} T_{V I} \Delta_{3}^{J} \Delta_{3}^{S} T_{V I I} T_{3}^{H D}
$$

where $T_{I} \ldots T_{V I I}$ are depending of each robot type specific design, $\Delta_{i}^{J}$ are depending of robot's joints elastic displacement and $\Delta_{i}^{S}$ is depending of robot's links elastic displacement (Marinescu \& Nicolescu 2010).

The interest point of present paper being focused on joints, the study consists on developing a set of user-friendly numerical applications for determining the $\Delta_{i}^{J}$ terms, making a case study on a real gantry robot.

\section{NUMERICAL APPLICATIONS FOR P-JOINT ELASTIC BEHAVIOUR ANALYSIS}

Having the evaluation of prismatic joint elastic displacement as a purpose and aim of study $\left(\Delta_{i}^{J}\right.$ terms from above relation) the authors, using the mathematical model, developed in the paper (Nicolescu et al., 2010), determined the overall loading of the gantry robot reduced to corresponding axial and radial direction for each cam-follower and the specific loads on each cam-follower in accordance with specific design for each translational joint. The cam-followers that are used in guiding system of studied gantry robot, are constructive similar with a double row ball bearing and their internal elastic displacements are evaluated using numerical models developed by authors in a special-made software application, QBSA.

Result given by QBSA where used in a custom-made MathCAD application, made by authors, for determining the total elastic displacement of a prismatic joint subjected to loads.

2.1 Numerical application for determining the loads on axial and radial direction for each cam-follower

Based on mathematical model developed and explained in a previous paper (Marinescu \& Nicolescu 2010), the authors developed a numerical application for determining loads on each guiding point.

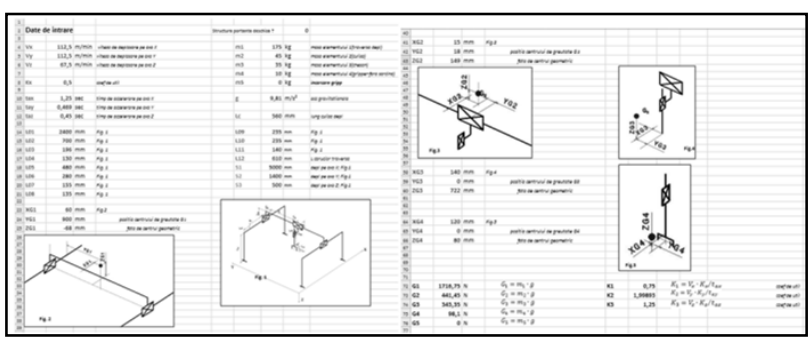

Fig. 1.Input data for numerical application for determining the loads cam-followers

The input data for application are the parameters at which the robot is operating, the structural dimensions and also the load that is manipulated by the robot. Also, for evaluating the inertial effects given by robot's axis, the loads and gravity centres were determined as shown in (Nicolescu \& Marinescu, 2010). For a bigger use domain, the application was design to accommodate also to opened gantry structures.

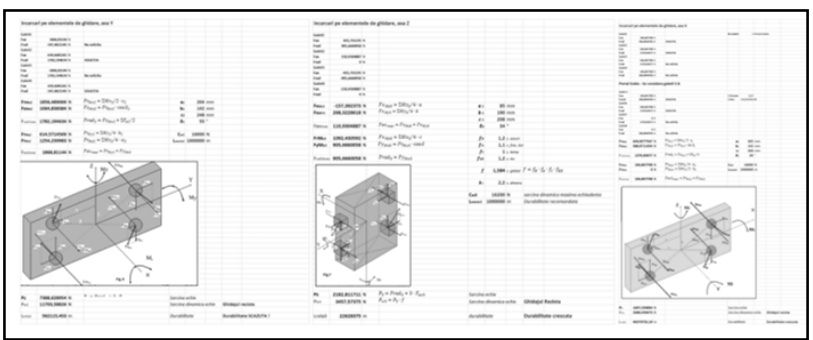

Fig. 2.Output data from numerical application for determining the loads cam-followers

Output results, as can be seen in the figure above, consists in numerical data that represents the values of load forces and their direction on each cam-follower from each one of the 
robot's prismatic joints, also the most load subjected camfollower is determined and it's fatigue life estimated.

\subsection{Analysis models for cam-followers internal elastic displacements evaluation}

Internal elastic displacements that occur on guiding system, as was mentioned in the begging of the paper, have great impact on robot's accuracy. Because of the construction similarities between cam-followers and bearings, the authors used as analysis platform the QBSA software which is dedicated for bearings behaviour evaluation.

With the usage of former obtained data, loads on axial and radial direction for each cam-follower, analysis models were developed for each guiding element.

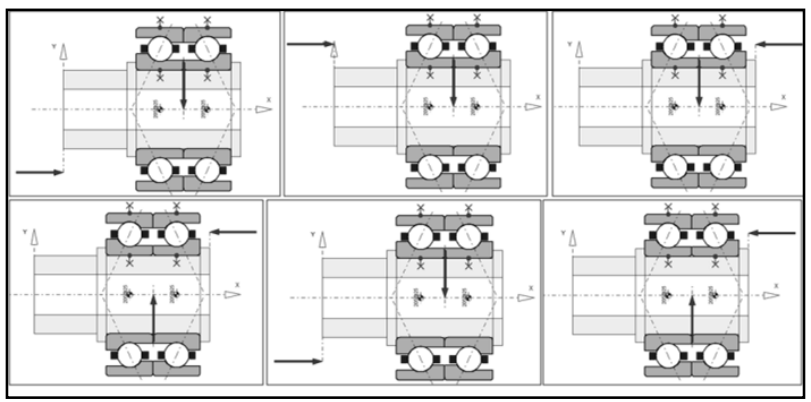

Fig. 3.Analysis models for cam-followers internal elastic displacements evaluation

With the above analysis models been created, the results representing internal elastic displacements, rings deformation and loads on each rolling body, were generated as can be seen in figure 4.

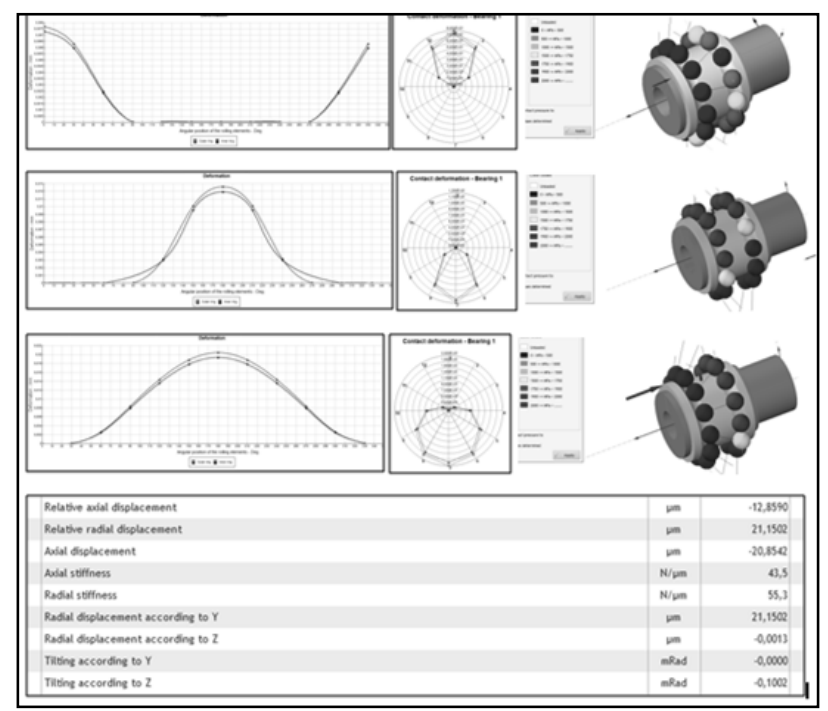

Fig. 4. Cam-followers internal displacement evaluation

\subsection{Analysis application for determining the total elastic} displacement of a P-Joint

The $\Delta_{i}{ }^{J}$ terms from equation (1), which are expressing the joint elastic displacements, have the following expression:

$$
\left[\Delta_{i}^{J}\right]=\left[\begin{array}{cccc}
n_{x} & o_{x} & a_{x} & p_{x} \\
n_{y} & o_{y} & a_{y} & p_{y} \\
n_{z} & o_{z} & a_{z} & p_{z} \\
0 & 0 & 0 & 1
\end{array}\right]
$$

The mathematical model for determining the total elastic displacement corresponding to a prismatic joint was developed by authors in a previous paper (Marinescu D. \& Nicolescu A, 2010) and for the study involved in present paper was developed a custom-made MathCAD application for determining the values of axial and angular displacements.

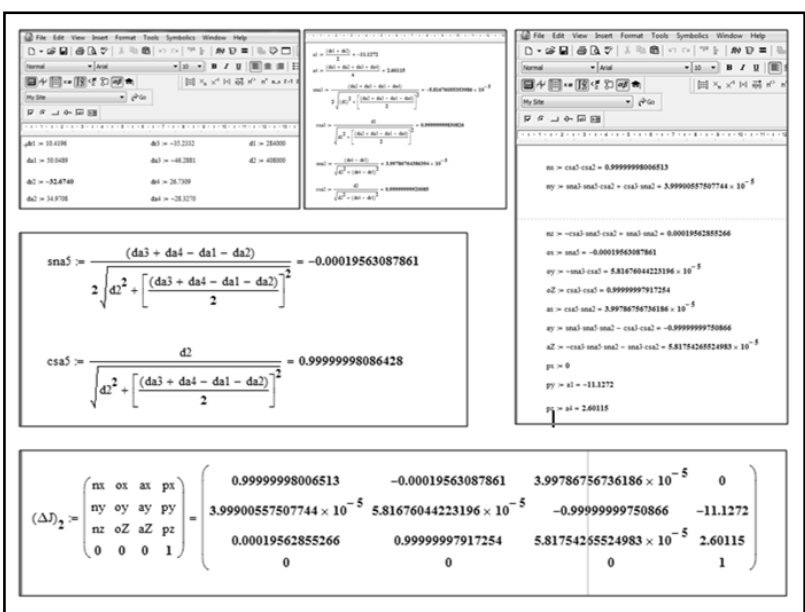

Fig. 5.Total elastic displacement evaluation for a prismatic joint

Using data collected above, the authors determined the numerical values for joints displacements that are used on a real gantry robot. Combining the results on each joint, the error inducted in robot's accuracy was determined, thus making a case study on an industrial robot from Gudel manufacturer.

\section{CONCLUSION}

This work came as set of practically numerical applications that were developed having as mathematical background the research made by authors having as a subject the elastic displacement within gantry robots joints. The aim being to make a set of tools that came to help in the process of designing linear axis, components sets configuration and developing online and off-line programming strategies for gantry robots.

Study case was made on a Gudel robot for which, by using presented applications, optimum guiding system configuration was determined corresponding to a particular type of industrial application.

In order to have the entire area of study covered, the authors are intending to develop analysis and numerical applications for determining structure elements elastic deformation influence on gantry robot's accuracy.

\section{ACKNOWLEDGEMENTS}

The work has been funded by the Sectoral Operational Programme Human Resources Development 2007-2013 of the Romanian Ministry of Labour, Family and Social Protection through the Financial Agreement POSDRU/88/1.5/S/60203.

\section{REFERENCES}

Marinescu, D.; Nicolescu, A. (2010). Gantry robot volumetric error evaluation using analytical and FEM modelling, Proceedings of DAAAM 2010, 20-23rd Oct 2010, Zadar, Croatia, ISSN 1726-9679, ISBN 978-3-901509-73-5, Katalinic, B. (Ed.), pp. 1059, Published by DAAAM International, Vienna, 2010

Nicolescu, A.; Marinescu, D. \& Ivan, M. (2010). Elastic displacement influence of translation joints on volumetric accuracy for gantry industrial robots (Part1\&2), Proceedings OPTIROB 2010, Olaru, A.; Ciupitu L. (Ed.), pp. 28-32, ISBN: 978-981-08-5840-7, Calimanesti,

Nicolescu A., Stanciu M (1996). Elastic Behavior Modeling of Industrial Robot's Base Translation Modules. Part.1. The Mathematical Model, Proceeding RAAD '96), pp. 239-243, ISBN: 963420482 1, Budapest Hungary, June 1996

*** (2009) http://www.gudel.com, Accessed on: 2011-04-22

*** (2010) http://www.bearinganalysis.com,, Accessed on: 2011-04-15 